\title{
Unique Range Set for Meromorphic Functions with Deficient Values
}

\author{
Abhijit Banerjee ${ }^{1}$ and Sujoy Majumder ${ }^{2}$ \\ ${ }^{1}$ Department of Mathematics, University of Kalyani, Kalyani, Nadia, West Bengal 741235, India \\ ${ }^{2}$ Department of Mathematics, Katwa College, Burdwan 713130, India
}

Correspondence should be addressed to Abhijit Banerjee; abanerjee_kal@yahoo.co.in

Received 13 May 2013; Accepted 10 August 2013

Academic Editor: Yan Xu

Copyright (C) 2013 A. Banerjee and S. Majumder. This is an open access article distributed under the Creative Commons Attribution License, which permits unrestricted use, distribution, and reproduction in any medium, provided the original work is properly cited.

We employ the idea of weighted sharing of sets to find a unique range set for meromorphic functions with deficient values. Our result improves, generalises, and extends the result of Lahiri. Examples are exhibited that a condition in one of our results is the best possible one.

\section{Introduction Definitions and Results}

In this paper by meromorphic functions we will always mean meromorphic functions in the complex plane. It will be convenient to let $E$ denote any set of positive real numbers of finite linear measure, not necessarily the same at each occurrence. For any nonconstant meromorphic function $h(z)$ we denote by $S(r, h)$ any quantity satisfying

$$
S(r, h)=o(T(r, h)) \quad(r \longrightarrow \infty, r \notin E) .
$$

We denote by $T(r)$ the maximum of $T(r, f)$ and $T(r, g)$. The notation $S(r)$ denotes any quantity satisfying $S(r)=$ $o(T(r))$ as $r \rightarrow \infty, r \notin E$.

We adopt the standard notations of the Nevanlinna theory of meromorphic functions as explained in [1]. For $a \in \mathbb{C} \cup\{\infty\}$, we define

$$
\Theta(a, f)=1-\limsup _{r \rightarrow \infty} \frac{\bar{N}(r, a ; f)}{T(r, f)} .
$$

Let $f$ and $g$ be two nonconstant meromorphic functions, and let $a$ be a finite complex number. We say that $f$ and $g$ share $a$ CM, provided that $f-a$ and $g-a$ have the same zeros with the same multiplicities. Similarly, we say that $f$ and $g$ share $a$ IM, provided that $f-a$ and $g-a$ have the same zeros ignoring multiplicities. In addition we say that $f$ and $g$ share $\infty \mathrm{CM}$, if $1 / f$ and $1 / g$ share $0 \mathrm{CM}$, and we say that $f$ and $g$ share $\infty$ IM, if $1 / f$ and $1 / g$ share 0 IM.

Let $S$ be a set of distinct elements of $\mathbb{C} \cup\{\infty\}$ and $E_{f}(S)=$ $\bigcup_{a \in S}\{z: f(z)=a\}$, where each point is counted according to its multiplicity. Denote by $\bar{E}_{f}(S)$ the reduced form of $E_{f}(S)$. If $E_{f}(S)=E_{g}(S)$, we say that $f$ and $g$ share the set $S$ CM. On the other hand if $\bar{E}_{f}(S)=\bar{E}_{g}(S)$, we say that $f$ and $g$ share the set $S$ IM. Evidently, if $S$ contains only one element, then it coincides with the usual definition of CM (resp., IM) shared values.

Let a set $S \subset \mathbb{C}$ and $f$ and $g$ be two nonconstant meromorphic (entire) functions. If $E_{f}(S)=E_{g}(S)$ implies $f \equiv g$, then $S$ is called a unique range set for meromorphic (entire) functions or in brief URSM (URSE). We will call any set $S \subset \mathbb{C}$ a unique range set for meromorphic functions ignoring multiplicity (URSM-IM) for which $\bar{E}_{f}(S)=\bar{E}_{g}(S)$ implies $f \equiv g$ for any pair of nonconstant meromorphic functions.

Inspired by Nevanlinna's 5 and 4 value theorem, in [2,3], Gross raised the problem of finding out a finite set $S$ so that an entire function in the complex plane is determined by the preimage of $S$, where each pre-image of $S$ related to some entire function is counted according to its multiplicity.

In 1982 Gross and Yang [4] proved the following theorem.

Theorem A. Let $S=\left\{z \in \mathbb{C}: e^{z}+z=0\right\}$. If two entire functions $f$ and $g$ satisfy $E_{f}(S)=E_{g}(S)$, then $f \equiv g$.

Noting that the set $S$ in Theorem A is an infinite set, we know that Theorem A does not give a solution to the problem of Gross.

In $1994 \mathrm{Yi}$ [5] exhibited a URSE with 15 elements, and in $1995 \mathrm{Li}$ and Yang [6] exhibited a URSM with 15 elements and a URSE with 7 elements. Since then, the quest for minimum 
cardinality for a URSM created an increasing interest among the researchers.

By now the URSM with 11 elements is the smallest available URSM obtained by Frank and Reinders [7]. It is also seen that the finite URSMs are the set of distinct zeros of some suitable polynomials.

A polynomial $P$ in $\mathbb{C}$ is called a strong uniqueness polynomial for meromorphic (entire) functions if, for any nonconstant meromorphic (entire) functions $f$ and $g, P(f) \equiv c P(g)$ implies $f \equiv g$, where $c$ is a suitable nonzero constant. We say that $P$ is SUPM (SUPE) in brief. On the other hand for a polynomial $P$ in $\mathbb{C}$, if the condition $P(f) \equiv P(g)$ implies $f \equiv g$ for any nonconstant meromorphic (entire)s function $f$ and $g$, then $P$ is called a uniqueness polynomial for meromorphic (entire) functions. We say that $P$ is a UPM (UPE) in brief.

Suppose that $P$ is a polynomial of degree $n$ in $\mathbb{C}$ having only simple zeros and that $S$ is the set of all zeros of $P$. If $S$ is a URSM (URSE), then from the definition it follows that $P$ is UPM (UPE). However the converse is not, in general, true. For $P(z)=a z+b(a \neq 0)$ is clearly a UPM, but for $f=$ $-(b / a) e^{z}$ and $g=-(b / a) e^{-z}$ we see that $E_{f}(S)=E_{g}(S)$, where $S=\{-b / a\}$ is the set of zeros of $P(z)=a z+b$.

To find under which condition the converse is true Fujimoto [8] first invented a special property of a polynomial, which he called the property $(H)$. Fujimoto's property $(H)$ may be stated as follows: a polynomial $P$ is said to satisfy the property $(H)$ if $P(\alpha) \neq P(\beta)$ for any two distinct zeros $\alpha, \beta$ of the derivative $P^{\prime}$.

Fujimoto found a sufficient condition for a set of zeros $S$ of a SUPM (SUPE) $P$ to be a URSM (URSE) as follows.

Theorem B (see [8]). Let $P$ be polynomial of degree $n$ in $\mathbb{C}$ having only simple zeros satisfying the condition $(H)$. Let $P^{\prime}$ have $k$ distinct zeros, and let either $k \geq 3$ or $k=2$ and $P^{\prime}$ have no simple zero. Further suppose that $P$ is a SUPM (SUPE). If S is the set of zeros of $P$ and $n \geq 2 k+7(n \geq 2 k+3)$, then $S$ is a URSM (URSE).

Answers to the question of Gross $[2,3]$ and the analogous question for meromorphic functions on $\mathbb{C}$ were given by Yi [9] and Li and Yang [6,10] who investigated the zero sets of polynomials $P$ of the form

$$
P(z)=z^{n}+a z^{n-m}+b,
$$

where $n>m \geq 1$ and $a$ and $b$ are so chosen so that $P$ has $n$ distinct roots. When $m \geq 2$, then there is no problem regarding property $(H)$ as in [11] it has been shown that the zero set $S$ of $P(z)$ is a URSM, and hence $P(z)$ is a UPM. But whenever $m=1, P(z)$ is not satisfying property $(H)$ as here $P^{\prime}(z)$ has two distinct zeros and one of them is simple. So natural question would be whether, for $m=1$, the zero set $S$ of $P(z)$ can be a URSM or even be a URSE.

In this direction some investigations were already done independently by both Yi [9] and Li and Yang [6] for entire functions. The following result was proved by them.
Theorem C. Let $S=\left\{z: z^{7}-z^{6}-1=0\right\}$. If $f$ and $g$ are two nonconstant entire functions satisfying $E_{f}(S)=E_{g}(S)$, then $f \equiv g$.

Clearly $z^{7}-z^{6}-1$ is a UPE. So it will be interesting to investigate the analogous results for meromorphic functions for more general polynomial, namely, $z^{n}+a z^{n-1}+b$. In this perspective in 1996 Yi proved the following theorem.

Theorem D (see [11]). Let $S=\left\{z: z^{n}+a z^{n-1}+b=0\right\}$, where $n(\geq 11)$ is an integer and $a$ and $b$ are two nonzero constants such that the algebraic equation $z^{n}+a z^{n-1}+b=0$ has no multiple roots. If $f$ and $g$ are nonconstant meromorphic functions satisfying $E_{f}(S)=E_{g}(S)$, then either $f \equiv$ gor $f=-\left(a h\left(h^{n-1}-\right.\right.$ $1)) /\left(h^{n}-1\right), g=-\left(a\left(h^{n-1}-1\right)\right) /\left(h^{n}-1\right)$, where $h=f / g$.

Theorem D is not much significant as it do not commensurate with the aforesaid discussion. That is to say under the supposition of Theorem D, S cannot be a URSM.

In the meantime Fang and Hua [12] extended Theorem C to meromorphic functions with the help of some additional conditions on the ramification indexes of $f$ and $g$. Fang and Hua [12] proved the following theorem.

Theorem E (see [12]). Let $S$ be defined as in Theorem C. If two meromorphic functions $f$ and $g$ are such that $\Theta(\infty, f)>$ $11 / 12, \Theta(\infty, g)>11 / 12$, and $E_{f}(S)=E_{g}(S)$, then $f \equiv g$.

We now require the following definition known as weighted sharing of sets and values which renders a useful tool for the purpose of relaxation of the nature of sharing the sets.

Definition 1 (see $[13,14]$ ). Let $k$ be a nonnegative integer or infinity. For $a \in \mathbb{C} \cup\{\infty\}$ one denotes by $E_{k}(a, f)$ the set of all $a$ points of $f$, where an $a$-point of multiplicity $m$ is counted $m$ times if $m \leq k$ and $k+1$ times if $m>k$. If $E_{k}(a, f)=E_{k}(a, g)$, one says that $f$ and $g$ share the value $a$ with weight $k$.

We write that $f$ and $g$ share $(a, k)$ to mean that $f$ and $g$ share the value $a$ with weight $k$. Clearly if $f$ and $g$ share $(a, k)$, then $f$ and $g$ share $(a, p)$ for any integer $p, 0 \leq p<k$. Also we note that $f, g$ share a value $a$ IM or CM if and only if $f$ and $g$ share $(a, 0)$ or $(a, \infty)$, respectively.

Definition 2 (see [13]). Let $S$ be a set of distinct elements of $\mathbb{C} \cup\{\infty\}$ and $k$ a nonnegative integer or $\infty$. One denotes by $E_{f}(S, k)$ the set $E_{f}(S)=\bigcup_{a \in S}\{z: f(z)-a=0\}$. Clearly $E_{f}(S)=E_{f}(S, \infty)$ and $\bar{E}_{f}(S)=E_{f}(S, 0)$.

Definition 3 (see [15]). For $a \in \mathbb{C} \cup\{\infty\}$ one denotes by $N(r, a$; $f \mid=1$ ) the counting function of simple $a$-points of $f$. For a positive integer $m$ one denotes by $N(r, a ; f \mid \leq m)(N(r, a$; $f \mid \geq m)$ ) the counting function of those $a$-points of $f$ whose multiplicities are not greater (less) than $m$, where each $a$ point is counted according to its multiplicity.

$\bar{N}(r, a ; f \mid \leq m)(\bar{N}(r, a ; f \mid \geq m))$ are defined similarly, where in counting the $a$-points of $f$ we ignore the multiplicities. 
Also $N(r, a ; f<m), N(r, a ; f>m), \bar{N}(r, a ; f<m)$, and $\bar{N}(r, a ; f \mid>m)$ are defined analogously.

We define

$$
\delta_{2}(a, f)=1-\limsup _{r \rightarrow \infty} \frac{N_{2}(r, a ; f)}{T(r, f)},
$$

where $N_{2}(r, a ; f)=\bar{N}(r, a ; f)+\bar{N}(r, a ; f \mid \geq 2)$.

Lahiri [16] improved Theorem $\mathrm{E}$ in the following direction.

Theorem $\mathbf{F}$ (see [16]). Let $\mathbf{S}$ be defined as in Theorem C. If, for two nonconstant meromorphic functions $f$ and $g, \Theta(\infty, f)+$ $\Theta(\infty, g)>3 / 2$ and $E_{f}(S, 2)=E_{g}(S, 2)$, then $f \equiv g$.

In 2004 Lahiri and Banerjee [17] further improved Theorem $\mathrm{C}$ in a more compact and convenient way and obtained the following result.

Theorem G (see [17]). Let $S=\left\{z: z^{n}+a z^{n-1}+b=0\right\}$, where $n(\geq 9)$ is an integer and $a, b$ are two nonzero constants such that $z^{n}+a z^{n-1}+b=0$ has no multiple root. If $E_{f}(S, 2)=$ $E_{g}(S, 2)$ and $\Theta(\infty, f)+\Theta(\infty, g)>4 /(n-1)$, then $f \equiv g$.

So we observe that deficiencies of poles play a vital role in order to find the sufficient condition for which the conclusion of Theorems D and F-G holds well. The first motivation of the paper is to show that even the deficiencies of other values contribute significantly for the conclusion of Theorems F and G. We now give the following example which establishes the fact that the set $S$ in Theorems F-G can not be replaced by any arbitrary set containing six distinct elements.

Example 4. Let $f(z)=\sqrt{\alpha \beta \gamma} e^{z}, g(z)=\sqrt{\alpha \beta \gamma} e^{-z}$, and $S=$ $\{\alpha \sqrt{\beta}, \alpha \sqrt{\gamma}, \beta \sqrt{\alpha}, \beta \sqrt{\gamma}, \gamma \sqrt{\alpha}, \gamma \sqrt{\beta}\}$, where $\alpha, \beta$, and $\gamma$ are three nonzero distinct complex numbers. Clearly $E_{f}(S, \infty)=$ $E_{g}(S, \infty)$, but $f \neq \equiv g$.

So it remains an open problem for investigations whether the degree of the equation defining $S$ in Theorem G can be reduced to six. This is the second motivation of writing the paper. In short we will further improve, generalize, and extend Theorems F and G.

The following theorem is the main result of the paper.

Theorem 5. Let $S=\left\{z: z^{n}+a z^{n-1}+b=0\right\}$, where $n(\geq 6)$ is an integer and $a, b$ are two nonzero constants such that $z^{n}+$ $a z^{n-1}+b=0$ has no multiple roots. Suppose that $f$ and $g$ are two nonconstant meromorphic functions satisfying $E_{f}(S, m)=$ $E_{g}(S, m)$. If

(i) $m \geq 2$ and $\Theta_{f}+\Theta_{g}>\max \{(9-n) / 2,(n+1) /(n-1)\}$

(ii) orm $=1$ and $\Theta_{f}+\Theta_{g}>\max \{(10-n) / 2,(n+1) /(n-1)\}$

(iii) orm $=0$ and $\Theta_{f}+\Theta_{g}>\max \{(15-n) / 2,(n+1) /(n-1)\}$,

then $f \equiv g$, where $\Theta_{f}=\Theta(0, f)+\Theta(\infty, f)+(1 / 2) \delta_{2}(-a, f)$ and $\Theta_{g}$ can be similarly defined.
The following examples show that the condition $\Theta_{f}+$ $\Theta_{g}>(n+1) /(n-1)$ is sharp in Theorem 5 when $n \geq 7$ and $m \geq 2$.

Example 6 (Example 2, [17]). Let $f=-a\left(\left(1-h^{n-1}\right) /\left(1-h^{n}\right)\right)$ and $g=-a h\left(\left(1-h^{n-1}\right) /\left(1-h^{n}\right)\right)$, where $h=\left(\left(\alpha^{2}\left(e^{z}-1\right)\right) /\left(e^{z}-\right.\right.$ $\alpha)), \alpha=\exp (2 \pi i / n)$, and $n(\geq 3)$ is an integer.

Then $T(r, f)=(n-1) T(r, h)+O(1), T(r, g)=(n-1)$ $T(r, h)+O(1)$, and $T(r, h)=T\left(r, e^{z}\right)+O(1)$. Further we see that $h \neq \alpha, \alpha^{2}$ and so for any complex number $\gamma \neq \alpha, \alpha^{2}$, $\bar{N}(r, \gamma ; h) \sim T(r, h)$. We also note that a root of $h=1$ is neither a pole nor a zero of $f$ and $g$. Hence $\Theta(\infty, f)=$ $\Theta(\infty, g)=2 /(n-1)$. On the other hand $\Theta(0, f)=$ $1-\lim \sup _{r \rightarrow \infty}\left(\left(\sum_{k=1}^{n-2} \bar{N}\left(r, \beta^{k} ; h\right)+\bar{N}(r, \infty ; h)\right) /((n-1)\right.$ $T(r, h)+O(1)))=0$ and $\Theta(0, g)=1-\lim _{\sup } \rightarrow \infty\left(\left(\sum_{k=1}^{n-2} \bar{N}(r\right.\right.$, $\left.\left.\left.\beta^{k} ; h\right)+\bar{N}(r, 0 ; h)\right) /((n-1) T(r, h)+O(1))\right)=0$, where $\beta=$ $\exp (2 \pi i /(n-1))$. Also $\delta_{2}(-a, f)=1-\lim _{\sup } \rightarrow \infty((2 \bar{N}(r$, $0 ; h)) /((n-1) T(r, h)+O(1)))=(n-3) /(n-1)$ and $\delta_{2}(-a, f)=$ $1-\lim \sup _{r \rightarrow \infty}((2 \bar{N}(r, \infty ; h)) /((n-1) T(r, h)+O(1)))=$ $(n-3) /(n-1)$. Therefore $\Theta_{f}+\Theta_{g}=(n+1) /(n-1)$. Clearly $E_{f}(S, \infty)=E_{g}(S, \infty)$ because $f^{n-1}(f+a) \equiv g^{n-1}(g+a)$, but $f \not \equiv g$.

Example 7. Let $f$ and $g$ be given as in Example 6, where $h=$ $\left(\left(\alpha\left(\alpha e^{z}-1\right)\right) /\left(e^{z}-1\right)\right), \alpha=\exp (2 \pi i / n)$, and $n(\geq 3)$ is an integer.

We now explain some definitions and notations which are used in the paper.

Definition 8 (see [18]). Let $f$ and $g$ be two nonconstant meromorphic functions such that $f$ and $g$ share $(a, 0)$. Let $z_{0}$ be an $a$-point of $f$ with multiplicity $p$, an $a$-point of $g$ with multiplicity $q$. One denotes by $\bar{N}_{L}(r, a ; f)$ the reduced counting function of those $a$-points of $f$ and $g$, where $p>q$, by $N_{E}^{1)}(r$, $a ; f)$ the counting function of those $a$-points of $f$ and $g$, where $p=q=1$, and by $\bar{N}_{E}^{(2}(r, a ; f)$ the reduced counting function of those $a$-points of $f$ and $g$, where $p=q \geq 2$. In the same way one can define $\bar{N}_{L}(r, a ; g), N_{E}^{1)}(r, a ; g)$, and $\bar{N}_{E}^{(2}(r$, $a ; g)$. In a similar manner one can define $\bar{N}_{L}(r, a ; f)$ and $\bar{N}_{L}$ $(r, a ; g)$ for $a \in \mathbb{C} \cup\{\infty\}$.

When $f$ and $g$ share $(a, m), m \geq 1$, then $N_{E}^{1)}(r, a ; f)=$ $N(r, a ; f \mid=1)$.

Definition 9. One denotes by $\bar{N}(r, a ; f \mid=k)$ the reduced counting function of those $a$-points of $f$ whose multiplicities are exactly $k$, where $k \geq 2$ is an integer.

Definition 10 (see $[13,14])$. Let $f, g$ share a value $a$ IM. One denotes by $\bar{N}_{*}(r, a ; f, g)$ the reduced counting function of those $a$-points of $f$ whose multiplicities differ from the multiplicities of the corresponding $a$-points of $g$.

Clearly $\bar{N}_{*}(r, a ; f, g) \equiv \bar{N}_{*}(r, a ; g, f)$ and $\bar{N}_{*}(r, a ; f, g)=$ $\bar{N}_{L}(r, a ; f)+\bar{N}_{L}(r, a ; g)$. 


\section{Lemmas}

In this section we present some lemmas which will be needed in the sequel. Let $F$ and $G$ be two nonconstant meromorphic functions defined as follows:

$$
F=\frac{f^{n-1}(f+a)}{-b}, \quad G=\frac{g^{n-1}(g+a)}{-b} .
$$

Henceforth we will denote by $H$ and $V$ the following two functions:

$$
H=\left(\frac{F^{\prime \prime}}{F^{\prime}}-\frac{2 F^{\prime}}{F-1}\right)-\left(\frac{G^{\prime \prime}}{G^{\prime}}-\frac{2 G^{\prime}}{G-1}\right) .
$$

Lemma 11 (see [19]). Let $f$ be a nonconstant meromorphic function, and let

$$
R(f)=\frac{\sum_{k=0}^{n} a_{k} f^{k}}{\sum_{j=0}^{m} b_{j} f^{j}}
$$

be an irreducible rational function in $f$ with constant coefficients $\left\{a_{k}\right\}$ and $\left\{b_{j}\right\}$, where $a_{n} \neq 0$ and $b_{m} \neq 0$. Then

$$
T(r, R(f))=d T(r, f)+S(r, f),
$$

where $d=\max \{n, m\}$.

Lemma 12 (see [18]). If $F$ and $G$ are two nonconstant meromorphic functions such that they share $(1,0)$ and $H \neq \equiv 0$, then

$$
\begin{aligned}
N_{E}^{1)}(r, 1 ; F \mid=1) & =N_{E}^{1)}(r, 1 ; G \mid=1) \\
& \leq N(r, H)+S(r, F)+S(r, G) .
\end{aligned}
$$

Lemma 13. Let $S=\left\{z: z^{n}+a z^{n-1}+b=0\right\}$, where $a$ and $b$ are nonzero constants such that $z^{n}+a z^{n-1}+b=0$ has no repeated root, $n(\geq 3)$ is an integer, and $F$ and $G$ are given by (5). If for two nonconstant meromorphic functions $f$ and $g E_{f}(S, 0)=$ $E_{g}(S, 0)$ and $H \neq 0$, then

$$
\begin{aligned}
N(r, H) \leq & \bar{N}(r, 0, f)+\bar{N}(r, 0 ; g) \\
& +\bar{N}(r, \infty ; f)+\bar{N}(r, \infty ; g)+\bar{N}(r,-a ; f \mid \geq 2) \\
& +\bar{N}(r,-a ; g \mid \geq 2)+\bar{N}_{*}(r, 1 ; F, G) \\
& +\bar{N}_{0}\left(r, 0 ; F^{\prime}\right)+\bar{N}_{0}\left(r, 0 ; G^{\prime}\right)
\end{aligned}
$$

where $\bar{N}_{0}\left(r, 0 ; F^{\prime}\right)$ is the reduced counting function of those zeros of $F^{\prime}$ which are not the zeros of $F(F-1)$, and $\bar{N}_{0}\left(r, 0 ; G^{\prime}\right)$ is similarly defined.

Proof. Since $E_{f}(S, 0)=E_{g}(S, 0)$, it follows that $F$ and $G$ share $(1,0)$. We can easily verify that possible poles of $H$ occur at (i) zeros of $f$ and $g$, (ii) multiple zeros of $f+a$ and $g+a$, (iii) poles of $f$ and $g$, (iv) those 1-points of $F$ and $G$ with different multiplicities, (v) zeros of $F^{\prime}$ which are not the zeros of $F(F-1)$, and (vi) zeros of $G^{\prime}$ which are not zeros of $G(G-1)$. Since $H$ has only simple poles, the lemma follows from above calculations. This proves the lemma.
Lemma 14 (see [17]). Let $f, g$ be two nonconstant meromorphic functions. Then $f^{n-1}(f+a) g^{n-1}(g+a) \neq \equiv b$, where $a$ and $b$ are nonzero finite constants, and $n(\geq 5)$ is an integer.

Lemma 15. Let $f, g$ be two nonconstant meromorphic functions such that $\Theta(0, f)+\Theta(\infty, f)+(1 / 2) \delta_{2}(-a, f)+\Theta(0, g)+$ $\Theta(\infty, g)+(1 / 2) \delta_{2}(-a, g)>(n+1) /(n-1)$; then $f^{n-1}(f+a) \equiv$ $g^{n-1}(g+a)$ implies $f \equiv g$, where $n(\geq 3)$ is an integer and $a$ is a nonzero finite constant.

Proof. Let

$$
f^{n-1}(f+a) \equiv g^{n-1}(g+a)
$$

And suppose $f \not \equiv g$. We consider two cases.

Case I. Let $y=g / f$ be a constant. Then from (11) it follows that $y \neq 1, y^{n-1} \neq 1, y^{n} \neq 1$, and $f \equiv-a\left(\left(1-y^{n-1}\right) /\left(1-y^{n}\right)\right)$, a constant, which is impossible.

Case II. Let $y=g / f$ be nonconstant. Then

$$
f \equiv-a \frac{1-y^{n-1}}{1-y^{n}} \equiv a\left(\frac{y^{n-1}}{1+y+y^{2}+\cdots+y^{n-1}}-1\right)
$$

From (2) we see by Lemma 11 that

$$
T(r, f)=(n-1) T(r, y)+S(r, y) .
$$

We first note that the zeros of $1+y+y^{2}+\cdots+y^{n-2}$ contribute to the zeros of both $f$ and $g$. In addition to this the poles of $y$ contribute to the zeros of $f$, and, since $g=f y$ the zeros of $y$ contribute to the zeros of $g$. So from (2) we see that

$$
\begin{gathered}
\sum_{j=1}^{n-2} \bar{N}\left(r, v_{j} ; y\right)+\bar{N}(r, \infty ; y) \leq \bar{N}(r, 0 ; f), \\
\sum_{k=1}^{n-1} \bar{N}\left(r, u_{k} ; y\right) \leq \bar{N}(r, \infty ; f),
\end{gathered}
$$

where $u_{k}=\exp (2 k \pi i / n)$ for $k=1,2, \ldots, n-1$ and $v_{j}=$ $\exp (2 j \pi i /(n-1))$ for $j=1,2, \ldots, n-2$.

Also since $n \geq 3$, from (2) we can find that $\bar{N}(r, 0 ; y) \leq$ $(1 / 2) N_{2}(r,-a ; f)$.

By the second fundamental theorem we get

$$
\begin{aligned}
(2 n-3) & T(r, y) \\
\leq & \bar{N}(r, \infty ; y)+\sum_{j=1}^{n-2} \bar{N}\left(r, v_{j} ; y\right)+\sum_{k=1}^{n-1} \bar{N}\left(r, u_{k} ; y\right) \\
& +\bar{N}(r, 0 ; y)+S(r, y)
\end{aligned}
$$




$$
\begin{aligned}
\leq & \bar{N}(r, 0 ; f)+\bar{N}(r, \infty ; f) \\
& +\frac{1}{2} N_{2}(r,-a ; f)+S(r, y) \\
\leq & \left(\frac{5}{2}-\Theta(0, f)-\Theta(\infty, f)-\frac{1}{2} \delta_{2}(-a, f)+\varepsilon\right) \\
& \times T(r, f)+S(r, y) \\
= & (n-1)\left(\frac{5}{2}-\Theta(0, f)-\Theta(\infty, f)-\frac{1}{2} \delta_{2}(-a, f)+\varepsilon\right) \\
& \times T(r, y)+S(r, y) .
\end{aligned}
$$

That is,

$$
\begin{aligned}
& \frac{2 n-3}{n-1} T(r, y) \\
& \leq\left(\frac{5}{2}-\Theta(0, f)-\Theta(\infty, f)-\frac{1}{2} \delta_{2}(-a, f)+\varepsilon\right) \\
& \quad \times T(r, y)+S(r, y),
\end{aligned}
$$

where $0<2 \varepsilon<\Theta(0, f)+\Theta(\infty, f)+\Theta(0, g)+\Theta(\infty, g)+$ $(1 / 2) \delta_{2}(-a, f)+(1 / 2) \delta_{2}(-a, g)$.

Again putting $y_{1}=1 / y$, noting that $T(r, y)=T\left(r, y_{1}\right)+$ $O(1)$, and proceeding in the same way as done in the above explanation we get that

$$
\begin{aligned}
& \frac{2 n-3}{n-1} T(r, y) \\
& \leq\left(\frac{5}{2}-\Theta(0, g)-\Theta(\infty, g)-\frac{1}{2} \delta_{2}(-a, g)+\varepsilon\right) \\
& \quad \times T(r, y)+S(r, y) .
\end{aligned}
$$

Adding (16) and (17) we get

$$
\begin{aligned}
& \left(\frac{4 n-6}{n-1}-5+\Theta(0, f)+\Theta(\infty, f)\right. \\
& +\frac{1}{2} \delta_{2}(-a, f)+\Theta(0, g)+\Theta(\infty, g) \\
& \left.+\frac{1}{2} \delta_{2}(-a, g)-2 \varepsilon\right) T(r, y) \\
& \quad \leq S(r, y)
\end{aligned}
$$

which is a contradiction.

Hence $f \equiv g$, and this proves the lemma.

Lemma 16 (see [20]). If $N\left(r, 0 ; f^{(k)} \mid f \neq 0\right)$ denotes the counting function of those zeros of $f^{(k)}$ which are not the zeros of $f$, where a zero of $f^{(k)}$ is counted according to its multiplicity, then

$$
\begin{aligned}
N\left(r, 0 ; f^{(k)} \mid f \neq 0\right) \\
\leq k \bar{N}(r, \infty ; f)+N(r, 0 ; f \mid<k) \\
\quad+k \bar{N}(r, 0 ; f \mid \geq k)+S(r, f) .
\end{aligned}
$$

\section{Proofs of the Theorems}

Proof of Theorem 5. We know from the assumption that the zeros of $z^{n}+a z^{n-1}+b$ are simple, and we denote them by $w_{j}$, $j=1,2, \ldots n$. Let $F, G$ be given by (5) and (6). Since $E_{f}(S, m)=E_{g}(S, m)$, it follows that $F$ and $G$ share $(1, m)$.

Case 1. If possible let us suppose that $H \neq \equiv$.

Subcase 1.1. Consider $m \geq 1$. While $m \geq 2$, using Lemma 16 we note that

$$
\begin{aligned}
\bar{N}_{0} & \left(r, 0 ; G^{\prime}\right)+\bar{N}(r, 1 ; G \mid \geq 2)+\bar{N}_{*}(r, 1 ; F, G) \\
\leq & \bar{N}_{0}\left(r, 0 ; G^{\prime}\right)+\bar{N}(r, 1 ; G \mid \geq 2)+\bar{N}(r, 1 ; G \mid \geq 3) \\
\leq & N\left(r, 0 ; G^{\prime} \mid G \neq 0\right)+S(r, g) \\
\leq & \bar{N}(r, 0 ; g)+\bar{N}(r,-a ; g) \\
& +\bar{N}(r, \infty ; g)+S(r, g) .
\end{aligned}
$$

Hence using (20) and Lemmas 11, 12 and 13 we get from the second fundamental theorem for $\varepsilon>0$ that

$$
\begin{aligned}
& (n+1) T(r, f) \\
& \leq \bar{N}(r, 0 ; f)+\bar{N}(r,-a ; f)+\bar{N}(r, \infty ; f) \\
& +N(r, 1 ; F \mid=1)+\bar{N}(r, 1 ; F \mid \geq 2) \\
& -N_{0}\left(r, 0 ; F^{\prime}\right)+S(r, f) \\
& \leq 2\{\bar{N}(r, 0 ; f)+\bar{N}(r, \infty ; f)\} \\
& +\bar{N}(r, 0 ; g)+\bar{N}(r, \infty ; g)+N_{2}(r,-a ; f) \\
& +\bar{N}(r,-a ; g \mid \geq 2)+\bar{N}(r, 1 ; G \mid \geq 2) \\
& +\bar{N}_{*}(r, 1 ; F, G)+\bar{N}_{0}\left(r, 0 ; G^{\prime}\right) \\
& +S(r, f)+S(r, g) \\
& \leq 2\{\bar{N}(r, 0 ; f)+\bar{N}(r, \infty ; f) \\
& +\bar{N}(r, 0 ; g)+\bar{N}(r, \infty ; g)\} \\
& +N_{2}(r,-a ; f) \\
& +N_{2}(r,-a ; g)+S(r, f)+S(r, g) \\
& \leq(10-2 \Theta(0, f)-2 \Theta(\infty, f)-2 \Theta(0, g) \\
& \left.-2 \Theta(\infty, g)-\delta_{2}(-a, f)-\delta_{2}(-a, g)+\varepsilon\right) T(r)+S(r) \\
& =\left(10-2 \Theta_{f}-2 \Theta_{g}+\varepsilon\right) T(r)+S(r) \text {, }
\end{aligned}
$$


In a similar way we can obtain

$$
(n+1) T(r, g) \leq\left(10-2 \Theta_{f}-2 \Theta_{g}+\varepsilon\right) T(r)+S(r) .
$$

Combining (21) and (3) we see that

$$
\left(n-9+2 \Theta_{f}+2 \Theta_{g}-\varepsilon\right) T(r) \leq S(r) .
$$

Since $\varepsilon>0$, (23) leads to a contradiction.

While $m=1$, using Lemma 16, (20) changes to

$$
\begin{aligned}
\bar{N}_{0}\left(r, 0 ; G^{\prime}\right)+\bar{N}(r, 1 ; G \mid \geq 2)+\bar{N}_{*}(r, 1 ; F, G) \\
\leq \bar{N}_{0}\left(r, 0 ; G^{\prime}\right)+\bar{N}(r, 1 ; G \mid \geq 2) \\
\quad+\bar{N}_{L}(r, 1 ; G)+\bar{N}(r, 1 ; F \mid \geq 3) \\
\leq N\left(r, 0 ; G^{\prime} \mid G \neq 0\right) \\
\quad+\frac{1}{2} \sum_{j=1}^{n}\left\{N\left(r, \omega_{j} ; f\right)-\bar{N}\left(r, \omega_{j} ; f\right)\right\} \\
\leq \bar{N}(r, 0 ; g)+\bar{N}(r,-a ; g)+\bar{N}(r, \infty ; g) \\
\quad+\frac{1}{2}\{\bar{N}(r, 0 ; f)+\bar{N}(r, \infty ; f)\}+S(r, f)+S(r, g) .
\end{aligned}
$$

So using (24) and Lemmas 12 and 13 and proceeding as in (21) we get from the second fundamental theorem for $\varepsilon>0$ that

$$
\begin{aligned}
(n+1) & T(r, f) \\
\leq & 2 \bar{N}(r, 0 ; f)+N_{2}(r,-a ; f)+2 \bar{N}(r, \infty ; f) \\
& +2 \bar{N}(r, 0 ; g)+N_{2}(r,-a ; g)+2 \bar{N}(r, \infty ; g) \\
& +\frac{1}{2}\{\bar{N}(r, 0 ; f)+\bar{N}(r, \infty ; f)\}+S(r, f)+S(r, g) \\
\leq & 2 \bar{N}(r, 0 ; f)+2 \bar{N}(r, \infty ; f)+N_{2}(r,-a ; f) \\
& +2 \bar{N}(r, 0 ; g)+2 \bar{N}(r, \infty ; g)+N_{2}(r,-a ; g) \\
& +T(r, f)+S(r, f)+S(r, g) \\
\leq & \left(11-2 \Theta_{f}-2 \Theta_{g}+\varepsilon\right) T(r)+S(r) .
\end{aligned}
$$

Similarly we can obtain

$$
(n+1) T(r, g) \leq\left(11-2 \Theta_{f}-2 \Theta_{g}+\varepsilon\right) T(r)+S(r) .
$$

Combining (25) and (3) we see that

$$
\left(n-10+2 \Theta_{f}+2 \Theta_{g}-\varepsilon\right) T(r) \leq S(r) .
$$

Since $\varepsilon>0$, (27) leads to a contradiction.
Subcase 1.2. Consider $m=0$. Using Lemma 16 we note that

$$
\begin{aligned}
\bar{N}_{0}( & \left.r, 0 ; G^{\prime}\right)+\bar{N}_{E}^{(2}(r, 1 ; F) \\
& +2 \bar{N}_{L}(r, 1 ; G)+2 \bar{N}_{L}(r, 1 ; F) \\
\leq & \bar{N}_{0}\left(r, 0 ; G^{\prime}\right)+\bar{N}_{E}^{(2}(r, 1 ; G) \\
& +\bar{N}_{L}(r, 1 ; G)+\bar{N}_{L}(r, 1 ; G)+2 \bar{N}_{L}(r, 1 ; F) \\
\leq & \bar{N}_{0}\left(r, 0 ; G^{\prime}\right)+\bar{N}(r, 1 ; G \mid \geq 2) \\
& +\bar{N}_{L}(r, 1 ; G)+2 \bar{N}_{L}(r, 1 ; F) \\
\leq & N\left(r, 0 ; G^{\prime} \mid g \neq 0\right)+\bar{N}(r, 1 ; G \mid \geq 2) \\
& +2 \bar{N}(r, 1 ; F \mid \geq 2) \\
\leq & \bar{N}(r, 0 ; g)+\bar{N}(r, \infty ; g)+\bar{N}(r,-a ; g) \\
& +\bar{N}(r, 0 ; g)+\bar{N}(r, \infty ; g) \\
& +2 \bar{N}(r, 0 ; f)+2 \bar{N}(r, \infty ; f)+S(r, f)+S(r, g) \\
\leq & \bar{N}(r, 0 ; g)+\bar{N}(r, \infty ; g)+\bar{N}(r,-a ; g) \\
& +2 T(r, g)+4 T(r, f)+S(r, f)+S(r, g) .
\end{aligned}
$$

Hence using (28) and Lemmas 12 and 13 we get from the second fundamental theorem for $\varepsilon>0$ that

$$
\begin{aligned}
(n+1) & T(r, f) \\
\leq & \bar{N}(r, 0 ; f)+\bar{N}(r,-a ; f)+\bar{N}(r, \infty ; f)+N_{E}^{1)}(r, 1 ; F) \\
& +\bar{N}_{L}(r, 1 ; F)+\bar{N}_{L}(r, 1 ; G) \\
& +\bar{N}_{E}^{(2}(r, 1 ; F)-N_{0}\left(r, 0 ; F^{\prime}\right)+S(r, f) \\
\leq & 2\{\bar{N}(r, 0 ; f)+\bar{N}(r, \infty ; f)\} \\
& +\bar{N}(r, 0 ; g)+\bar{N}(r, \infty ; g) \\
& +N_{2}(r,-a ; f)+\bar{N}_{(r,-a ; g l \geq 2)} \\
& +\bar{N}_{E}^{(2}(r, 1 ; F)+2 \bar{N}_{L}(r, 1 ; G) \\
& +2 \bar{N}_{L}(r, 1 ; F)+\bar{N}_{0}\left(r, 0 ; G^{\prime}\right)+S(r, f)+S(r, g) \\
\leq & 2\left\{\bar{N}_{(}(r, 0 ; f)+\bar{N}_{(}(r, \infty ; f)\right\} \\
+ & N_{2}(r,-a ; f)+2\{\bar{N}(r, 0 ; g)+\bar{N}(r, \infty ; g)\} \\
+ & N_{2}(r,-a ; g) \\
+ & 4 T(r, f)+2 T(r, g)+S(r, f)+S(r, g) \\
\leq & (16-2 \Theta f-2 \Theta g+\varepsilon) T(r)+S(r) . \\
&
\end{aligned}
$$


In a similar manner we can obtain

$$
(n+1) T(r, g) \leq\left(16-2 \Theta_{f}-2 \Theta_{g}+\varepsilon\right) T(r)+S(r) .
$$

Combining (29) and (3) we see that

$$
\left(n-15+2 \Theta_{f}+2 \Theta_{g}-\varepsilon\right) T(r) \leq S(r) .
$$

Since $\varepsilon>0$, (31) leads to a contradiction.

Case 2. Take $H \equiv 0$. On integration we get from (6)

$$
\frac{1}{F-1} \equiv \frac{A}{G-1}+B
$$

where $A$ and $B$ are constants and $A \neq 0$. From (32) we obtain

$$
F \equiv \frac{(B+1) G+A-B-1}{B G+A-B} .
$$

Clearly (33) together with Lemma 11 yields

$$
T(r, f)=T(r, g)+O(1) .
$$

Subcase 2.1. Suppose that $B \neq 0,-1$.

If $A-B-1 \neq 0$, from (33) we obtain

$$
\bar{N}\left(r, \frac{B+1-A}{B+1} ; G\right)=\bar{N}(r, 0 ; F) .
$$

From above calculations, Lemma 11, and the second fundamental theorem we obtain

$$
\begin{aligned}
n T(r, g)< & \bar{N}(r, \infty ; G)+\bar{N}(r, 0 ; G) \\
& +\bar{N}\left(r, \frac{B+1-A}{B+1} ; G\right)+S(r, g) \\
\leq & \bar{N}(r, \infty ; g)+\bar{N}(r, 0 ; g)+\bar{N}(r, 0 ; g+a) \\
& +\bar{N}(r, 0 ; f)+\bar{N}(r, 0 ; f+a)+S(r, g) \\
\leq & 2 T(r, f)+3 T(r, g)+S(r, g),
\end{aligned}
$$

which in view of (34) implies a contradiction as $n \geq 6$. Thus $A-B-1=0$, and hence (33) reduces to

$$
F \equiv \frac{(B+1) G}{B G+1}
$$

From this we have

$$
\bar{N}\left(r, \frac{-1}{B} ; G\right)=\bar{N}(r, \infty ; f) .
$$

Again by Lemma 11 and the second fundamental theorem we have

$$
\begin{aligned}
n T(r, g)< & \bar{N}(r, \infty ; G)+\bar{N}(r, 0 ; G) \\
& +\bar{N}\left(r, \frac{-1}{B} ; G\right)+S(r, g) \\
\leq & \bar{N}(r, \infty ; g)+\bar{N}(r, 0 ; g) \\
& +\bar{N}(r, 0 ; g+a)+\bar{N}(r, \infty ; f)+S(r, g) \\
\leq & T(r, f)+3 T(r, g)+S(r, g),
\end{aligned}
$$

which in view of (34) leads to a contradiction since $n \geq 6$.
Subcase 2.2. Suppose that $B=-1$.

From (33) we obtain

$$
F \equiv \frac{A}{-G+A+1}
$$

If $A+1 \neq 0$, from (40) we obtain

$$
\bar{N}(r, A+1 ; G)=\bar{N}(r, \infty ; f) .
$$

So using the same argument as used in the above subcase we can again obtain a contradiction. Hence $A+1=0$, and we have from (40) that $F G \equiv 1$ that means $f^{n-1}(f+a) g^{n-1}(g+$ a) $\equiv b^{2}$, which is impossible by Lemma 14 .

Subcase 2.3. Suppose that $B=0$.

From (33) we obtain

$$
F \equiv \frac{G+A-1}{A} .
$$

If $A-1 \neq 0$, from (42) we obtain

$$
\bar{N}(r, 1-A ; G)=\bar{N}(r, 0 ; F) .
$$

So in the same manner as above calculations we again get a contradiction. So $A=1$, and hence $F \equiv G$; that is, $f^{n-1}(f+$ $a) \equiv g^{n-1}(g+a)$. Now the theorem follows from Lemma 15 .

\section{Acknowledgments}

The authors wish to thank the referee for his/her valuable remarks and suggestions. Abhijit Banerjee is thankful to DSTPURSE programme for financial assistance.

\section{References}

[1] W. K. Hayman, Meromorphic Functions, Oxford Mathematical Monographs, Clarendon Press, Oxford, UK, 1964.

[2] F. Gross, "Factorization of meromorphic functions andsome open problems," in Proceedings of the Conference Held at the University of Kentucky, vol. 599 of Lecture Notes in Mathematics, Lexington, Ky, May 1976.

[3] F. Gross, "Factorization of meromorphic functions and some open problems," in Complex Analysis, vol. 599 of Lecture Notes in Mathematics, pp. 51-67, Springer, Berlin, Germany, 1977.

[4] F. Gross and C. C. Yang, "On preimage and range sets of meromorphic functions," Proceedings of the Japan Academy A, vol. 58, no. 1, pp. 17-20, 1982.

[5] H.X. Yi, “On a problem of Gross," Science in China A, vol. 24, pp. 1137-1144, 1994.

[6] P. Li and C.-C. Yang, "Some further results on the unique range sets of meromorphic functions," Kodai Mathematical Journal, vol. 18, no. 3, pp. 437-450, 1995.

[7] G. Frank and M. Reinders, "A unique range set for meromorphic functions with 11 elements," Complex Variables, vol. 37, no. 1-4, pp. 185-193, 1998.

[8] H. Fujimoto, "On uniqueness of meromorphic functions sharing finite sets," American Journal of Mathematics, vol. 122, no. 6, pp. 1175-1203, 2000. 
[9] H. X. Yi, "A question of Gross and the uniqueness of entire functions," Nagoya Mathematical Journal, vol. 138, pp. 169-177, 1995.

[10] P. Li and C.-C. Yang, "On the unique range set of meromorphic functions," Proceedings of the American Mathematical Society, vol. 124, no. 1, pp. 177-185, 1996.

[11] H.-X. Yi, "Unicity theorems for meromorphic or entire functions III," Bulletin of the Australian Mathematical Society, vol. 53, no. 1, pp. 71-82, 1996.

[12] M. Fang and X. Hua, "Meromorphic functions that share one finite set CM," Journal of Nanjing University, vol. 15, no. 1, pp. 15-22, 1998.

[13] I. Lahiri, "Weighted sharing and uniqueness of meromorphic functions," Nagoya Mathematical Journal, vol. 161, pp. 193-206, 2001.

[14] I. Lahiri, "Weighted value sharing and uniqueness of meromorphic functions," Complex Variables, vol. 46, no. 3, pp. 241-253, 2001.

[15] I. Lahiri, "Value distribution of certain differential polynomials," International Journal of Mathematics and Mathematical Sciences, vol. 28, no. 2, pp. 83-91, 2001.

[16] I. Lahiri, "A question of gross and weighted sharing of a finite set by meromorphic functions," Applied Mathematics E-Notes, vol. 2, pp. 16-21, 2002.

[17] I. Lahiri and A. Banerjee, "Uniqueness of meromorphic functions with deficient poles," Kyungpook Mathematical Journal, vol. 44, no. 4, pp. 575-584, 2004.

[18] H.-X. Yi, "Meromorphic functions that share one or two values II," Kodai Mathematical Journal, vol. 22, no. 2, pp. 264-272, 1999.

[19] A. Z. Mokhon'ko, "The Nevanlinna characteristics of certain meromorphic functions," Functional Analysis and Its Applications, no. 14, pp. 83-87, 1971.

[20] I. Lahiri and S. Dewan, "Value distribution of the product of a meromorphic function and its derivative," Kodai Mathematical Journal, vol. 26, no. 1, pp. 95-100, 2003. 


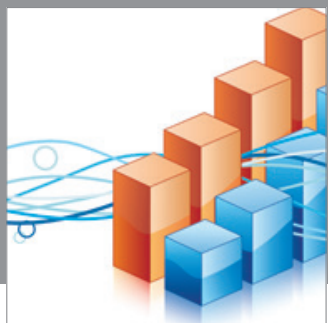

Advances in

Operations Research

mansans

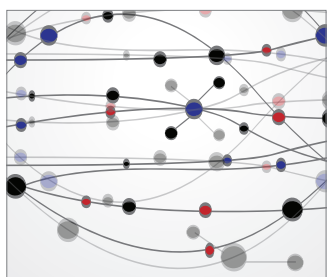

The Scientific World Journal
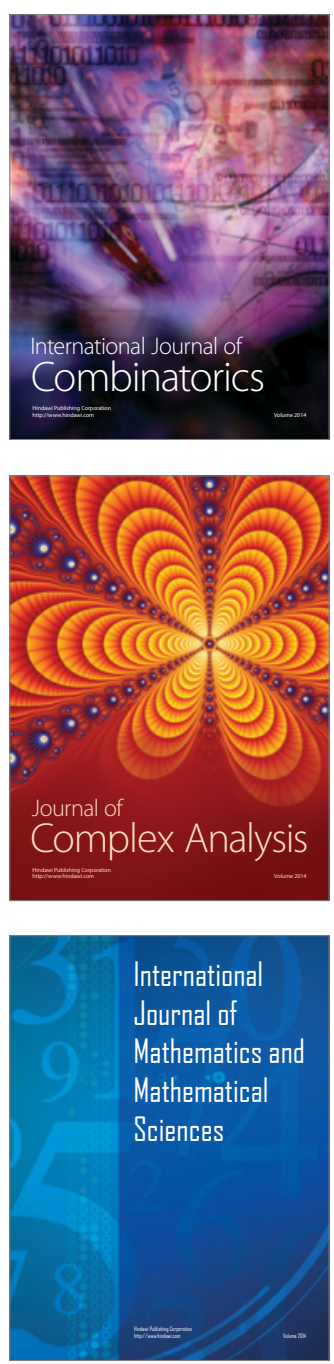
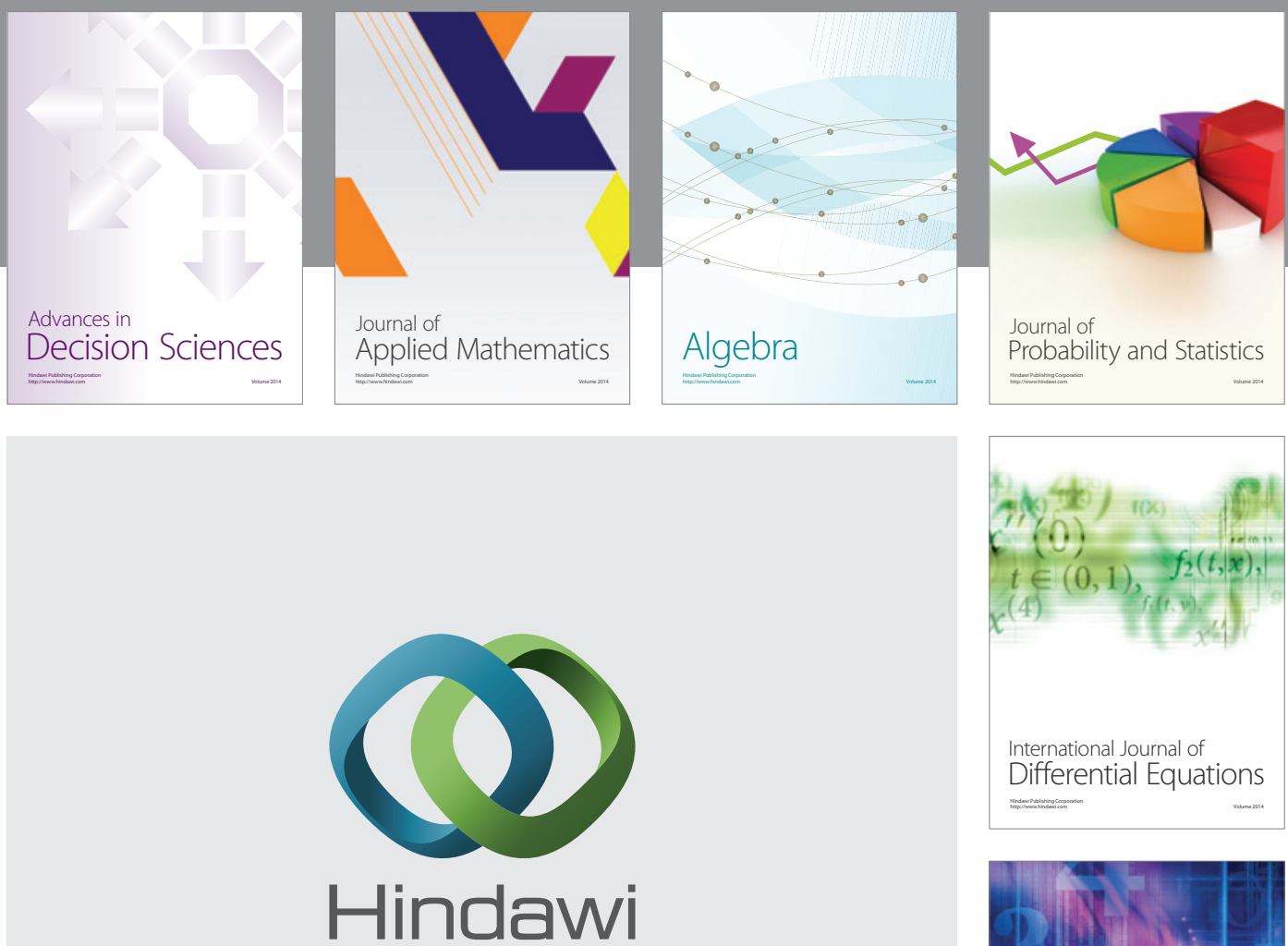

Submit your manuscripts at http://www.hindawi.com
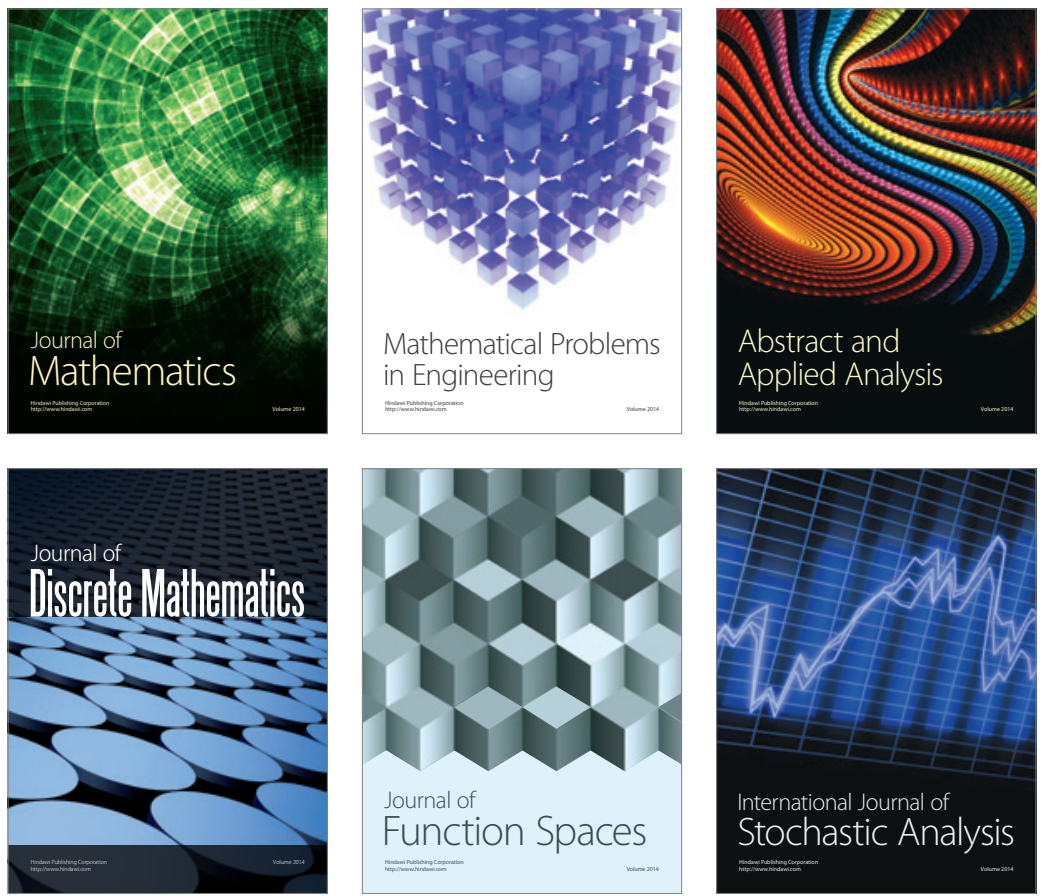

Journal of

Function Spaces

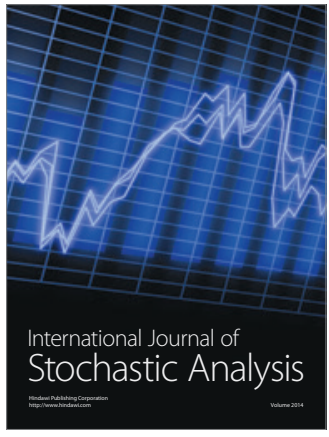

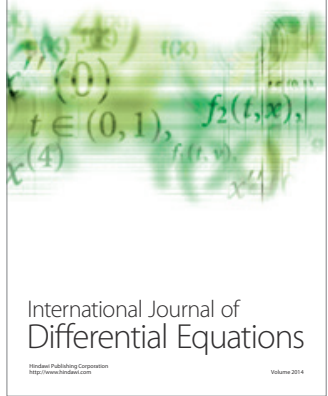
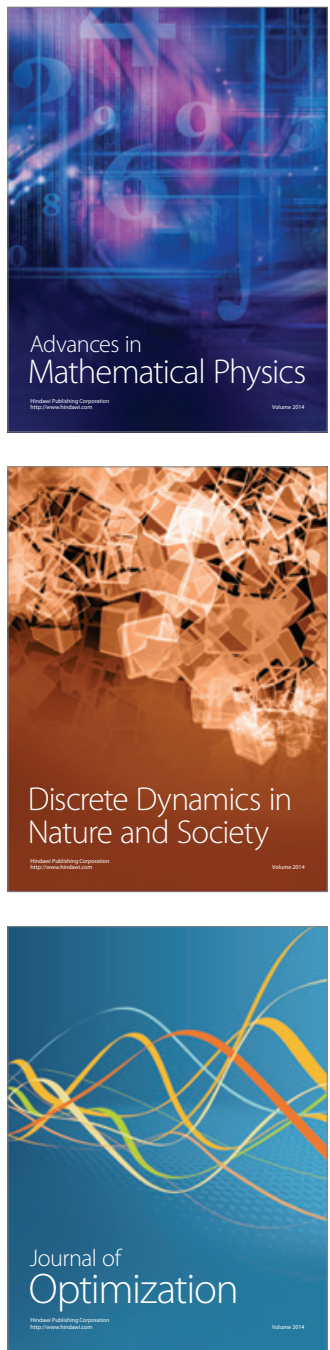